Review Article

\title{
Thrombophilia: how far should a clotter be investigated?
}

\author{
Isobel D. Walker
}

Thrombosis Research Group, Department of Haematology, Glasgow Royal Infirmary, Glasgow G4 05F, UK

\section{Introduction}

Virchow ${ }^{1}$ postulated that the key conditions promoting thrombosis are changes in the vessel wall (vascular injury), impairment of the blood flow (stasis), and alterations in the blood itself (hypercoagulability). The concept of a congenital deficiency of a single regulatory factor that could shift the haemostatic balance so significantly that the resultant hypercoagulability would cause clinical thrombosis seemed to be proven by the observation of the association of familial thrombosis with antithrombin deficiency. ${ }^{2}$ It has, however, become clear that the components of Virchow's triad are not independent - abnormality of one element may promote abnormalities in the others. Furthermore, abnormality of a single element may not lead to thrombosis unless abnormality of a second element supervenes - for example, individuals with inherited deficiency of the natural anticoagulant mechanisms may be at significant thrombotic risk only if vascular injury or stasis is superimposed. In a century and a half a great deal of experimental evidence has accrued to support Virchow's triad but in purely practical terms frequently it remains impossible to determine why an individual has suffered a thrombosis.

In the developed world, thromboembolism remains a major cause of mortality and morbidity. Since the long-term health and cost implications are enormous, prevention of thrombosis must be a priority. When a patient does present with thrombosis we must not only manage the acute event but ensure the risk of recurrence is minimized.

\section{Terminology}

The terminology used to describe conditions which may increase the risk of thrombosis is confused and there are no universally accepted definitions. The term thrombophilia has been used in the United Kingdom to describe 'the familial or acquired abnormalities of the haemostatic mechanism likely to predispose to thrombosis'. ${ }^{3}$ This defines

Correspondence: I.D. Walker, M.D., F.R.C.P., F.R.C.Path. Received: 15 December 1993 thrombophilia in laboratory terms but clinicians would normally restrict it to include only those defects shown to be associated with increased thrombotic risk in a significant proportion of affected individuals. Within this group are included the inherited abnormalities of anti-thrombin, protein $\mathrm{C}$ and protein $\mathrm{S}$, and acquired abnormalities such as lupus anticoagulants and anti-cardiolipins. Elsewhere authors define thrombophilia in clinical terms - for example, as 'a tendency toward venous thromboembolic disease in adults under 50 years old in the absence of known risk factors including, among others, malignancy, immobilization or major surgery'. ${ }^{4}$

The term hypercoagulability is somewhat broader and may be used to embrace not only thrombophilic defects as described above but any shift in the haemostatic balance which may promote inappropriate or excessive platelet/fibrin deposition without necessarily to date having been shown to be associated with clinical thromboembolism in a significant proportion of affected individuals. The term prothrombotic is yet wider still and may be used to describe any abnormality of any element of Virchow's triad. Thus conditions such as trauma, malignancy, myeloproliferation or nephrotic syndrome which do not primarily affect haemostasis are included along with primary hypercoagulable conditions.

Confusion has arisen from a tendency to use the words prothrombotic and prethrombotic interchangeably. The prefix 'pre' indicates an irreversible evolution to a given effect, that is, a patient who is prethrombotic must inevitably develop thrombosis. Patients who have a prothrombotic state have an increased tendency to thrombosis but may never develop a thrombus, that is, they may never transform to a prethrombotic state.

\section{Thrombophilic defects \\ Antithrombin deficiency}

Antithrombin (AT) is the major plasma inhibitor of thrombin and other serine proteases of the 
intrinsic coagulation pathway. The activity of AT is enhanced in the presence of heparin or of certain endothelial cell-surface heparan sulphate proteoglycans. The structure and function of AT and the organization of the gene have been described in detail, and are reviewed by Lane et al. and by Cooper. ${ }^{5,6}$

In accord with the classification of other coagulation protein deficiencies, AT deficiency characterized by a concomitant reduction in functional activity and protein antigen is called type 1 (classical) deficiency whilst those deficiencies in which functional activity and protein antigen are discordantly reduced (the protein antigen assay results being greater than the detectable functional activity) are called type 2. Many attempts to further subclassify AT deficiency are recorded but to date none has gained universal acceptance.

Inherited AT deficiency is associated with increased risk of thrombosis. The incidence of thromboembolic disease is highest in association with type 1 deficiency and type 2 defects affecting the reactive site. It has been suggested that heterozygous type 2 defects affecting the heparin binding site (HBS) may not increase thrombotic risk ${ }^{8,9}$ but certain type 2 HBS mutations may be clinically more important than others. ${ }^{10}$

The relative prevalences of type 1 and type 2 AT deficiencies have been unclear. In symptomatic cohorts they may be equally common ${ }^{11}$ but studies reporting large numbers of deficient kindred have given conflicting impressions. ${ }^{7,8}$ After screening 25,000 females, Abildgaard ${ }^{12}$ estimated the prevalence of type $1 \mathrm{AT}$ deficiency in the general population to be $1 / 5,000$. This prevalence of type 1 AT deficiency has been confirmed in a recent survey of 10,000 blood donors in which it was also demonstrated that the prevalence of type 2 AT deficiency is much greater and may be as high as $1 / 600-1 / 700 .^{13}$

A variety of immunological and functional assays for AT are commercially available. The distribution of AT activity is approximately normal and, although definite age- and sex-related variations have been reported, these are minor and a single fairly narrow reference range may be used. ${ }^{14}$ The identification of individuals with AT deficiency is therefore relatively simple.

\section{Protein C deficiency}

Protein $\mathrm{C}(\mathrm{PC})$ is a vitamin $\mathrm{K}$-dependent glycoprotein synthesized by the liver. On the endothelial cell surface, thrombin in the presence of thrombomodulin cleaves an arginine-leucine bond converting PC into activated protein C (APC). APC inactives the coagulation cofactors FVa and FVIIIa exerting regulatory activity on both intrinsic and extrinsic pathways. In addition APC neu- tralizes plasminogen activator inhibitor-1 (PAI-1) the major inhibitor of tissue plasminogen activator (t-PA) and thus enhances fibrinolytic activity. ${ }^{15-17}$ Both type 1 and type 2 deficiency are recognized. The genetic defects in a large number of type 1 and type 2 patients have been determined and recurrent mutations noted. ${ }^{18-21}$

In many families there appears to be a clear association between PC deficiency and a thrombotic tendency. ${ }^{22}$ It has been estimated that the prevalence of heterozygous PC deficiency associated with thrombosis is around $1 / 16,000$ to $1 / 32,000$ of the population. ${ }^{23,24}$ This figure is widely different from the observation that heterozygosity for PC deficiency affects as many as 1 in 200-300 healthy blood donors. ${ }^{25}$ Furthermore, although homozygotes or compound heterozygotes for PC deficiency may suffer from neonatal purpura fulminans and life-threatening venous thrombosis ${ }^{26,27}$ their heterozygous parents usually have no personal or family history of thrombosis. These observations have led to reconsideration of the hypothesis that heterozygous protein $\mathrm{C}$ deficiency is in itself enough to increase thrombotic risk substantially and it has recently been proposed that in symptomatic heterozygotes some other as yet unidentified factor(s) (genetic or acquired) may be contributing to the risk. ${ }^{28}$

The majority of known PC variants cause reduced function detectable in chromogenic assays but a small number of variants have been found that behave normally in chromogenic assays but abnormally in clotting assays. Protein $\mathrm{C}$ activity displays a log normal distribution and significant variation with age. ${ }^{29}$ Age-restricted reference ranges are therefore recommended when reporting PC activity results. Interpretation of PC results is complicated by the considerable overlap which exists between normals and heterozygotes for PC deficiency.

\section{Protein $S$ deficiency}

Protein S (PS) is a non-enzymatic vitamin Kdependent cofactor - essential for the expression of the anticoagulant function of APC. Hereditary deficiency of PS was first reported in $1984^{30,31}$ and the association of inherited PS deficiency with a tendency to develop thrombosis is well documented. ${ }^{32}$

Regulation of haemostasis may not be the sole function of PS since it forms non-covalent complexes with one of the complement system regulatory proteins $-\mathrm{C}_{4} \mathrm{~b}$ binding protein. Currently most diagnostic laboratories use ELISA-based assays to measure total PS antigen and free PS antigen. Over the past few years there have been many attempts to devise meaningful assays of PS 
activity but to date there is little consensus on how to perform a routine assay of PS activity.

The subclassification of PS deficiency has proven particularly complicated and the significance of reduced free PS antigen is difficult to interpret. The study of the molecular lesions responsible for PS defects is also problematic - hampered by the large size and exon number of the gene. There are no large studies reported of the influence of demographic factors on PS levels. The diagnosis of PS deficiency remains difficult in many instances. The prevalence of PS deficiency in the general population is unknown.

\section{Anti-phospholipid antibodies}

At one time believed to be the same, lupus anticoagulants and anti-cardiolipin antibodies are now considered different antibody types within the wide spectrum of anti-phospholipid antibodies (APAs). Anti-cardiolipin (aCL) antibodies bind to anionic phospholipids or phospholipid-containing complexes in solid phase immunoassays whereas lupus anticoagulant (LA) antibodies interfere with, and prolong phospholipid-dependent coagulation tests, such as the activated partial thromboplastin time (APTT), kaolin clotting (KCT) and dilute Russell viper venom time (dRVVT). Recently it has become clear that the interactions between APAs and their putative 'target' antigens may be more complex than previously thought and that these interactions may require the participation of cofactors. ${ }^{33-35}$

Anti-phospholipid antibodies may be found in patients with a variety of 'underlying' disorders including autoimmune disorders, malignancies, infections and in association with a number of drugs. Some patients develop APAs and clinical complications including thrombosis without any apparent underlying pathology. These patients have been considered to have 'primary antiphospholipid syndrome'. ${ }^{36}$

Many authors have described an association between aCL antibodies or LA antibodies and thromboembolic disease. ${ }^{37-40}$ Recruiting consecutive unselected systemic lupus erythematosus (SLE) patients and using objective tests to detect evidence of previous thrombosis, Long et al. ${ }^{41}$ demonstrated that SLE patients who had APAs detectable as both $\mathrm{aCL}$ and LA antibodies had a statistically significantly increased incidence of thrombosis, and that SLE patients who had only LA antibodies had a positive trend towards increased numbers of thrombotic events. At present the mechanism or mechanisms by which APAs play a pathogenetic role in thrombosis are unclear.

The recent explosion of interest in APAs has meant that routine haemostasis laboratories are now regularly asked to perform screening tests. At the same time there remains considerable controversy about the most appropriate methods to use. In screening for LA antibodies meticulous sample preparation is essential. Further, it is recommended that the APTT alone is an inadequate initial screen for LA antibodies and other phospholipid dependent tests, for example, the dRVVT and the KCT must be included. Once prolongation of the initial screening tests is shown to be due to the presence of an inhibitor, confirmation that the inhibitory activity is directed against phospholipid-containing complexes is mandatory. ${ }^{42}$ Kits for performing ELISA assays of anti-cardiolipins are widely available. Unfortunately at present there are no national or international standards available and results vary from laboratory to laboratory. In screening for APAs, it is necessary to include both clotting tests to detect LA antibodies and immunological assays of $\mathrm{aCL}$ antibodies.

\section{APC resistance}

Recently a new disorder which may be associated with familial thrombosis has been postulated. This disorder is characterized by a poor anticoagulant response (as measured by prolongation of an APTT) when APC is added to the patient's plasma ${ }^{43}$ It was hypothesized that the observed 'APC resistance' is most likely the result of deficiency in the patient's plasma of a second, as yet unidentified, cofactor for APC. However, more recently it has become evident that APC resistance may be the result of a molecular defect in factor $V .{ }^{44}$ The defect appears to be heritable and to cosegregate with an increased tendency to develop venous thrombosis.

A kit containing optimized APC and APTT reagents is available (Chromogenix, Sweden). Currently many haemostasis laboratories are evaluating this kit for routine use and screening their population of patients with unexplained thrombosis trying to determine the prevalence of 'APC resistance' in thrombotic patients and in the healthy population. ${ }^{45}$

\section{Fibrinolytic system abnormalities}

Although some abnormalities of fibrinogen molecules may increase the risk of thrombosis or arteriosclerosis $^{46}$ most dysfibrinogenaemias are detected by chance and are not associated with any clinical manifestations. Patients with both type 1 and type 2 plasminogen deficiency and a history of recurrent thrombosis are described ${ }^{47,48}$ but in affected families a low percentage of heterozygotes are symptomatic. ${ }^{49}$ Approximately $30 \%$ of patients who have had an idiopathic deep vein thrombosis have low baseline fibrinolytic activity or a 
diminished fibrinolytic response to a standardized venous occlusion test or to the infusion of desmopressin. The majority of poor responders have elevated PAI levels and only a minority $(10-20 \%$ of the poor responders) have reduced t-PA levels. ${ }^{50}$ Elevated PAI activity is found in association with a wide variety of clinical disorders but it has proven difficult to demonstrate a causal relationship with thrombosis. Furthermore the methodology for investigating the fibrinolytic system is cumbersome and not readily applicable to routine clinical investigation. Blood samples must be collected in a rigidly standardized fashion from fasting and resting patients. Of the stimulation tests, only venous occlusion is safe for the majority of patients, and this is a painful and often poorly tolerated procedure. The clinical relevance of these stimulation tests is questionable.

\section{Other abnormalities}

Individuals have been reported in whom thrombosis, venous or arterial, has been associated with a variety of other deficiencies, for example, heparin cofactor II, factor XII or cystathione- $\beta$-synthase. However, to date convincing family studies are lacking and screening for these abnormalities remains the province of specialist laboratories.

\section{Timing of investigation}

As far as possible, detailed investigation is best avoided in the acute post-thrombotic phase. Heparin therapy reduces plasma levels of functional AT and, because PC and PS are vitamin K-dependent, their levels are lowered by oral anticoagulants. Screening tests for LA antibodies and the APC resistance test are also unreliable in anticoagulated patients. Pregnancy and the contraceptive pill influence the levels of the natural anticoagulants thus, wherever possible, results should be confirmed when the patient is neither pregnant nor taking oral contraceptives.

\section{Detection of the prethrombotic state}

The processes which are responsible for converting the hypercoagulable prothrombotic state into a prethrombotic state and subsequently clinical thrombosis are poorly understood, but obviously it would be highly desirable to be able to detect this transition. The coagulation cascade is a series of linked proteolytic reactions. At each step an inactive zymogen is converted to an active serine protease which is then responsible for the subsequent zymogen to enzyme transition. The enzymes generated are very short lived in the circulation being rapidly neutralized by naturally occurring inhibitors. However, in the process of activation, peptides are released from the parent zymogens and these activation peptides are more stable entities with finite half-lives in the plasma.

Twenty years ago Nossel and co-workers ${ }^{51}$ developed an immunoassay of fibrinopeptide A (FPA), the peptide cleaved by thrombin from the $\alpha$ chain of fibrinogen. FPA levels are elevated in many clinical conditions and commercial kits for FPA assays have been developed. The clinical applicability of FPA assays is, however, limited by the high probability of in vitro thrombin activation. $^{52}$

Recently most interest has surrounded the detection and quantification of prothrombin fragment $1+2\left(F_{1+2}\right)$ released from the amino-terminal end of prothrombin when it is activated by factor Xa. Immunoenzymatic assays have been developed by commercial manufacturers and are widely available. Elevated $F_{1+2}$ levels have been reported in disseminated intravascular coagulation and in patients with venous thromboembolism..$^{53,54}$ In about $25 \%$ of asymptomatic and untreated patients with inherited thrombophilic defects, deficiency of AT, PC or PS, elevated FPA and/or $F_{1+2}$ levels have been recorded. $F_{1+2}$ levels are more frequently elevated in patients with $\mathrm{PC}$ or PS deficiency than in patients with AT deficiency. $F_{1+2}$ and FPA levels do not differ significantly between hereditary thrombophilic patients who have had a clinical history of thrombosis and those who have had no previous symptoms. ${ }^{55}$

\section{Investigation of patients with thrombosis}

In the current climate of concern about the cost of health care, the question of how far it is justifiable to take investigation of a patient with a history of thrombosis begs the response "what difference would knowing that a defect does or does not exist make to patient management?' Firstly, it is wortn emphasizing that objective diagnosis of thrombotic events - deep vein thrombosis (DVT) or pulmonary embolism (PE) - by radiology, ultrasound or impedance plethysmography, should be considered essential. It is rarely acceptable to diagnose thrombosis or embolism on clinical grounds alone. Secondly, diagnosing a modifiable prothrombotic pathology is evidently likely to be worthwhile. Thus the initial stages of investigation must be directed towards excluding or identifying common acquired causes of thrombosis - malignancy, myeloproliferative disease and so on - and routine haematology including a full blood count (FBC) and film, urea and electrolytes (U/E), liver function tests (LFTs) and plasma glucose levels are appropriate (Table I). 
Table I All patients with possible venous thrombosis merit

1. Objective diagnosis of DVT/PE

2. Detailed clinical history and examination to identify or exclude underlying pathology

3. Routine laboratory investigation including FBC and film, coagulation screen (APTT, PT, TT) U/E, LFTs and plasma glucose

Several years ago, in a guidelines document, the British Society for Haematology suggested an initial screen for thrombophilia should include functional assays of AT, PC and plasminogen, immunological assays of total and free PS, and tests for LA and aCL antibodies. ${ }^{3}$ Preceded by a coagulation screen (APTT, prothrombin time (PT) and thrombin clotting time (TT)) this protocol is aimed at detecting the commonest inherited and acquired thrombophilic defects, and identifying for further investigation patients who may have fibrinogen or factor XII abnormalities. Since publication of these guidelines, information about thrombophilia and about the various investigations used to detect defects has grown steadily.

It has become clear that the tests and their interpetation are more complex than they at first seemed. Detailed recommendations for performing lupus anticoagulant tests have been published. ${ }^{42,56}$ The interpretation of PC assays is complicated by the considerable overlap between normals and heterozygous PC-deficient patients. No widely accepted PS functional assay is available and the interpretation of reduced free PS results is difficult. It is best to avoid collecting samples for thrombophilia screening tests whilst the patient is in the acute post-thrombotic phase. The only exception to this general rule would be the patient with severe life- or limb-threatening thrombosis who is proving difficult to anticoagulate, and in whom the question of AT deficiency and possible AT replacement therapy has been raised. Otherwise screening for specific thrombophilic defects when the patient has a current thrombosis is unlikely to contribute anything useful to his immediate management. This, taken in conjunction with the undoubted difficulties in performing and interpreting tests, may mean that thrombophilia screening should be confined to laboratories with a particular interest.

Is it worthwhile organizing patient follow-up for thrombophilia screening at a later date? Data on the prevalences of thrombophilic defects in patients who have had thrombotic events are confused. Most studies report prevalence rates in 'young' patients with histories of spontaneous venous thromboembolism but differences in patient selection and diagnostic criteria have resulted in reported prevalence rates of $10-30 \% .{ }^{57-60}$ The diagnosis of an underlying haemostatic defect is potentially important not only for the patient himself but, in the case of the heritable defects, for his family. Detection of APAs should initiate further investigation to exclude or identify treatable pathology underlying their development. Perhaps in patients in whom it has been decided on clinical grounds that long-term anticoagulation is mandatory, striving to diagnose an inherited thrombophilic defect may be viewed by some as pointless. However, in these patients, as in patients who have an already identified heritable thrombophilic defect, screening of family members is important to identify affected but as yet asymptomatic relatives.

At present the additional information which may be gained from extending testing to subclassify inherited defects and define genetic variants is interesting but rarely useful in making clinical decisions in patients who have already had thrombosis. In only a very few patients, consideration may be given to extending investigation to include fibrinolytic system assessment and assays of other defects which may occasionally be associated with thrombosis (Table II). Since heterozygous plasminogen is now believed not to be strongly associated with increased thrombotic risk a functional assay of plasminogen need no longer be included as part of an initial thrombophilia screen but may be included at this stage.

Recently Griffin et al. ${ }^{4}$ reported that the newly described defect in anticoagulant response to APC was present in $52-64 \%$ of plasmas from patients with a history of venous thrombosis with no other identified predisposing cause. If these findings are corroborated, the chances of finding explanations for many more thrombotic events will be greatly enhanced.

There are no clear guidelines on the management of patients with thrombophilia and it remains difficult to give advice. Asymptomatic relatives and thrombophilic patients who have already had a thrombotic event but are not on long-term anticoagulant prophylaxis constitute a considerable challenge. Management is aimed at preventing the initial or recurrent events but at present is largely empiric since the currently available tests for detecting the prethrombotic state are insufficiently sensitive to predict thrombosis.

How far is it justifiable to pursue specialized investigation of a patient with a history of thrombosis? Young patients under the age of $45^{3}$ and perhaps all patients up to age 70 or more ${ }^{60}$ who have had even a single episode of thrombosis either apparently spontaneously or in circumstances which in the great majority of individuals do not result in thrombosis (for example, pregnancy, contraceptive pill taking or long journeys) should be offered screening for thrombophilic defects 
(Table III). Screening tests should be aimed at detecting the most common defects - deficiency of AT, PC or PS, presence of LA or aCL antibodies, and APC resistance (Table IV). Finding an abnormality may only rarely influence the management of an acute event, but should in all cases highlight the requirement for increased vigilance and careful counselling in the patient's follow-up. In the case of heritable defects it is frequently not the patient himself who gains most from the diagnosis having been established but his affected relatives who are as yet asymptomatic.
Table III Who merits detailed thrombophilia screening?

1. Patients who have had one or more venous thrombotic event, and in whom detailed clinical and routin laboratory investigation has failed to reveal underlying pathology

2. Patients who have had venous thrombosis associated with exposure to minor increased thrombotic risk only, for example, pregnancy, contraceptive pill taking, long journeys, etc.

3. Relatives of patients known to have a heritable thrombophilic defect

Table IV Thrombophilia screening tests

Table II Additional tests - at present, unlikely to influence management and/or limited to research groups

1. DNA analysis, for example, AT, PC or PS

2. Fibrinolytic system tests

3. Other assays including heparin cofactor II, cystathione $\beta$ synthase

4. Assays of coagulation activation markers - FPA, $F_{1+2}$, thrombin - antithrombin (TAT)

\section{References}

1. Virchow, R. Ein Vortraguber die Thrombose von Jahre 1945. In: Gesammelte Abhandlungen zur Wissenschlaftlichen Medizin. Meidinger Sohn U. Co, Frankfurt, 1856, pp. 478-481.

2. Egeberg, $O$. Inherited antithrombin III deficiency causing thrombophilia. Thromb Diathes Haemorrhagica 1965, 13: $516-530$

3. British Society for Haematology. Guidelines on the investigation and management of thrombophilia. J Clin Pathol 1990, 43: 703-710.

4. Griffin, J.H., Evatt, B., Widerman, C. \& Fernandes, J.A. Anticoagulant protein $C$ pathway defective in majority of thrombophilic patients. Blood 1993, 82: 1989-1993.

5. Lane, D.A., Ireland, H., Olds, R.J., Perry, D.J. \& Aiach, M. Antithrombin III: a database of mutations. Thromb Haemostas 1991, 66: 657-661.

6. Cooper, D.N. The molecular genetics of familial venous thrombosis. Blood Rev 1991, 5: 55-70.

7. Lane, D.A., Old, R.J., Boisclair, M. et al. Antithrombin III mutation database: first update. Thromb Haemostas 1993, 70: 361-369.

8. Finazzi, G., Caccia, R. \& Barbui, T. Different prevalence of thromboembolism in the subtypes of congenital antithrombin III deficiency: review of 404 cases. Throm Haemostas 1987, 58: 1094.

9. Hultin, M.B., McKay, J. \& Abildgaard, U. Antithrombin Oslo: Type 1b classification of the first reported antithrombin-deficient family with a review of the hereditary antithrombin variants. Thromb Haemostas 1988, 59: 468-473.

10. Mohlo-Sabatier, P., Aiach, M., Gaillard, J. et al. Molecular characterisation of antithrombin III (ATIII) variants using polymerase chain reaction. Identification of the ATIII Charleville as an Ala 384-Pro mutation. J Clin Invest 1989, 84: 1236-1241.
Antithrombin activity

Protein C activity

Protein $\mathrm{S}$ antigen

Activated protein $\mathrm{C}$ resistance test

Lupus anticoagulant (LA) screen*

Anti-cardiolipins (aCLs)

Chromogenic assay Chromogenic assay ELISA total antigen ELISA free antigen

APTT

KCT

dRVVT

ELISA IgG aCL ELISA IgM aCL

*At least 2 of the LA screening tests should be done and if appropriate antiphospholipid activity confirmed with platelet neutralization.

11. Harper, P.L., Luddington, R.J., Daly, M. et al. The incidence of dysfunctional antithrombin variants: four cases in 210 patients with thromboembolic disease. Br J Haem 1991, 77 $360-364$.

12. Abildgaard, U. Antithrombin and related inhibitors of coagulation. In: Poller, L. (ed.) Recent Advances in Blood Coagulation. Churchill Livingstone, Edinburgh, 1981, pp. $151-173$.

13. Tait, R.C., Walker, I.D., Perry, D.J. et al. Prevalence of antithrombin III deficiency in the healthy population. $B r J$ Haem 1994 (in press).

14. Tait, R.C., Walker, I.D. \& Islam, S.I.A.M. Influence of demographic factors on antithrombin III activity in a healthy population. Br J Haematol 1993, 84: 476-486.

15. Stenflo, J. The biochemistry of protein C. In: Bertina, R.M (ed.) Protein $C$ and Related Proteins. Churchill Livingstone, Edinburgh, 1988, pp. 21-54.

16. Esmon, C.T. The roles of protein $\mathrm{C}$ and thrombomodulin in the regulation of blood coagulation. J Biol Chem 1989, 264: 4743-4746.

17. de Fouw, N.J., de Jong, Y.F., Haverkate, K. \& Bertina, R.M Activated protein $\mathrm{C}$ increases fibrin clot lysis by neutralisation of plasminogen activator inhibitor: no evidence for a cofactor role of protein S. Thromb Haemostas 1988, 60: 328-333.

18. Reitsma, P.H., Poort, S.R., Allaart, C.F., Briet, E. \& Bertina R.M. The spectrum of genetic defects in a panel of 40 Dutch families with symptomatic protein C deficiency type 1 : heterogeneity and founder effects. Blood 1991, 78: 890-894.

19. Grundy, C.B., Schulman, S., Krawczak, M., Kobosko, J., Kakkar, V.V. \& Cooper, D.N. Protein C deficiency and thromboembolism: recurrent mutation at Arg 306 in the protein C gene. Human Genet 1992, 88: 586-588. 
20. Bovill, E.G., Tomczak, J.A., Grant, B. et al. Protein C Vermont: symptomatic type II protein $\mathrm{C}$ deficiency associated with two Gla domain mutations. Blood 1992, 79: 1456-1465.

21. Reitsma, P.H., Poort, S.R., Bernardi, F. et al. Protein C deficiency: a database of mutations. Thromb Haemostas 1993, 69: 77-84.

22. Broekmans, A.W., Veltkamp, J.J. \& Bertina, R.M. Congenital protein $\mathrm{C}$ deficiency and venous thromboembolism. A study of three Dutch families. $N$ Engl J Med 1983, 309: $340-344$.

23. Gladson, C.L., Scharrer, I., Hach, V., Beck, K.H. \& Griffin, J.H. The frequency of type 1 heterozygous protein $S$ and protein $C$ deficiency in 141 unrelated young patients with venous thrombosis. Thromb Haemostas 1988, 59: 18-22.

24. Broekmans, A.W., van der Linden, I.K., Veltkamp, J.J. \& Bertina, R.M. Prevalence of isolated protein C deficiency in patients with venous thrombotic disease and in the population. Thromb Haemostas 1983, 50: 350.

25. Miletich, J., Sherman, L. \& Broze, G. Absence of thrombosis in subjects with heterozygous protein C deficiency. $N$ Engl $J$ Med 1987, 317: 991-996.

26. Seligsohn, U., Berger, A., Abend, M. et al. Homozygous protein $\mathrm{C}$ deficiency manifested by massive venous thrombosis in the newborn. $N$ Engl J Med 1984, 310: 559-569.

27. Marciniak, E., Wilson, H.D. \& Marlar, R.A. Neonatal purpura fulminans: a genetic disorder related to the absence of protein C in blood. Blood 1985, 65: 15-20.

28. Miletich, J.M., Prescott, S.M., White, R., Majerus, P.W. \& Bovill, E.G. Inherited predisposition to thrombosis. Cell 1993, 72: 477-480.

29. Tait, R.C., Walker, I.D., Islam, S.I.A.M. et al. Protein C activity in healthy volunteers - influence of age, sex, smoking and oral contraceptives. Thromb Haemostas 1993, 70: 281-285.

30. Comp, P.C., Nixon, R.R., Cooper, M.R. \& Esmon, C.T Familial protein $\mathbf{S}$ deficiency is associated with recurrent thrombosis. J Clin Invest 1984, 74: 2082-2088.

31. Schwarz, H.P., Fischer, M., Hopmeier, P., Batard, M.A. \& Griffin, J.H. Plasma protein $\mathbf{S}$ deficiency in familial thrombotic disease. Blood 1984, 64: 1297-1300.

32. Engesser, L., Broekmans, A.W., Briet, E., Brommer, E.J.P. \& Bertina, R.M. Hereditary protein S deficiency: clinical manifestations. Ann Intern Med 1987, 106: 677-682.

33. Bevers, E.M. \& Galli, M. $\beta_{2}$-Glycoprotein I for binding of anticardiolipin antibodies to cardiolipin. Lancet 1990, 336: 1952-1953.

34. McNeil, H.P., Simpson, R.J., Chesterman, C.N. \& Krilis, S.A. Anticardiolipin antibodies are directed against a complex antigen that includes a lipid-binding inhibitor of coagulation $\boldsymbol{\beta}_{2}$-Glycoprotein I (apoliprotein H). Proc Natl Acad Sci USA 1990, 87: 4120-4124.

35. Bevers, E.M., Galli, M., Barbui, T., Comfurius, P. \& Zwaal, R.F.A. Lupus anticoagulant IgG's (LA) are not directed to phospholipids only, but to a complex of lipid-bound human prothrombin. Thromb Haemostas 1991, 66: 629-632.

36. Hughes, G.R.V. The antiphospolipid syndrome: ten years on Lancet 1993, 342: 341-344.

37. Lechner, K. \& Pazbwinger-Fasching, I. Lupus anticoagulants and thrombosis. A study of 25 cases and review of literature. Haemostasis 1983, 15: 254-262.

38. Harris, E.N., Jaharvi, A.E., Boey, M.L. et al. Anticardiolipin antibodies: detection by radioimmunoassay and association with thrombosis in systemic lupus erythematosus. Lancet 1983, 2: $1211-1214$

39. Elias, M. \& Eldor, A. Thromboembolism in patients with 'lupus type' circulating anticoagulant. Arch Intern Med 1984, 144: $510-515$.

40. Love, P.E. \& Santoro, S.A. Antiphospholipid antibodies: anticardiolipin and the lupus anticoagulant in systemic lupus erythematosus (SLE) and in non-SLE disorders. Ann Intern Med 1990, 112: 682-698.
41. Long, A.A., Ginsberg, J.S., Brill Edwards, P. et al. The relationship of antiphospholipid antibodies to thromboembolic disease in systemic lupus erythematosus: a cross sectional study. Thromb Haemostas 1991, 66: 520-524.

42. BCSH Haemostasis and Thrombosis Task Force. Guidelines on testing for lupus anticoagulant. J Clin Pathol 1991, 44: 885-889.

43. Dahlback, B., Carlsson, M. \& Svensson, P.J. Familial thrombophilia due to a previously unrecognised mechanism characterised by poor anticoagulant response to activated protein C: prediction of a cofactor to activated protein C. Proc Natl Acad Sci USA 1993, 90: 1004.

44. Bauer, K.A. Hypercoagulability - a new cofactor in the protein C anticoagulant pathway. $N$ Engl $J$ Med 1994, 330: $566-567$.

45. Koster, T., Rosendaal, F.R., de Ronde, H., Briet, E., Vandenbroucke, J.P. \& Bertina, R.M. Venous thrombosis due to poor anticoagulant response to activated protein $\mathrm{C}$ : Leiden Thrombophilia Study. Lancet 1993, 342: 1503-1506.

46. Bithell, T.C. Hereditary dysfibrinogenaemia. Clin Chem 1985, 31: $509-516$

47. Hach Wunderle, V., Scharrer, I. \& Lottenberg, R. Congenital deficiency of plasminogen and its relationship to venous thrombosis. Thromb Haemostas 1988, 59: 277-280.

48. Scharrer, I.M., Wohl, R.C., Hach, V., Sinio, L., Boreisha, I. \& Robbins, K.C. Investigation of a congenital abnormal plasminogen Frankfurt 1 and its relationship to thrombosis. Thromb Haemostas 1986, 5: 396-401.

49. Dolan, G. \& Preston, F.E. Familial plasminogen deficiency and thromboembolism. Fibrinolysis 1988, 2 (Suppl 2): 26-34.

50. Juhan Vague, I., Valadier, J., Alessi, M.C. et al. Deficient tPA release and elevated PA inhibitor levels in patients with spontaneous or recurrent deep vein thrombosis. Thromb Haemostas 1987, 57: 67-72.

51. Nossel, H.L., Yudelman, I., Candield, R.E. et al. Measurement of fibrinopeptide A in human blood. J Clin Invest 1974 , 54: $43-53$

52. Owen, J. The utility of plasma fibrinopeptide assays. Thromb Haemostas 1989, 62: 807-810.

53. Bauer, K.A. \& Rosenberg, D. Thrombi generation in acute promyelocytic leukaemia. Blood 1984, 64: 791.

54. Bauer, K.A. \& Rosenberg, R.D. The pathophysiology of the prethrombotic state in humans: insights gained from studies using markers of the haemostatic system activation. Blood 1987, 70: 343-350.

55. Mannucci, P.M., Tripodi, A., Bottasso, B. et al. Markers of procoagulant imbalance in patients with inherited thrombophilic syndromes. Thromb Haemostas 1992, 67: 200-202.

56. SSC Subcommittee for the Standardisation of Lupus Anticoagulants. Guidelines for testing and revised criteria for lupus anticoagulants. Thromb Haemostas 1991, 65: 320-322.

57. Pabinger, I., Brucker, S., Kyrle, P.A. et al. Hereditary deficiency of antithrombin III, protein $C$ and protein $S$ : prevalence in patients with a history of venous thrombosis and criteria for rational patient screening. Blood Coagul Fibrinolysis 1992, 3: 547.

58. Ben-Tal, O., Zivelin, A. \& Seligsohn, U. The relative frequency of hereditary thrombotic disorders among 107 patients with thrombophilia in Israel. Thromb Haemostas 1989, 61: 50.

59. Malm, J., Laurell, M., Nilsson, I.M. \& Dahlback, B. Thromboembolic disease - critical evaluation of laboratory investigation. Thromb Haemostas 1992, 68: 7-13.

60. Melissari, E., Monte, G., Lindo, V.S. et al. Congenital thrombophilia among patients with venous thromboembolism. Blood Coag Fibrinol 1992, 3: 749-758. 